\title{
TeleWound care - providing remote wound assessment and treatment in the home care setting: current status and future directions
}

REVIEW

\author{
Nick Santamaria' \\ Suzanne Kapp ${ }^{2}$ \\ 'University of Melbourne and \\ Melbourne Health, Royal Melbourne \\ Hospital, Melbourne, VIC, Australia; \\ ${ }^{2}$ Royal District Nursing Service \\ Institute, Melbourne, VIC, Australia
}

Correspondence: Nick Santamaria University of Melbourne and Melbourne Health, Level 6, Royal Melbourne Hospital, Grattan Street, Parkville, Melbourne, VIC 3050, Australia

Tel +6I 4 I456 0929

Email nick.santamaria@mh.org.au
This article was published in the following Dove Press journal:

Smart Homecare Technology and TeleHealth

18 November 2013

Number of times this article has been viewed

\begin{abstract}
The use of wound telemedicine systems in the home care environment has been expanding for the last decade. These systems can generally be grouped into two main types: store and forward systems and video conference type systems; additionally, there are also hybrid systems available that include elements of both. Evidence to date suggests that these systems provide significant benefits to patients, clinicians, and to the health care system generally. Reductions in resource use, visit substitution, costs, and high patient and clinician satisfaction have been reported; however, there is a lack of integration with existing health care technology and no clearly defined technical or clinical standards as yet. Similarly, the legalities associated with wound telemedicine and remote consultation remain unclear. As wound telemedicine systems continue to evolve and be deployed in different locations, there remains significant potential to harness their power to benefit patients being treated at home.
\end{abstract}

Keywords: telemedicine, home care, e-health

\section{Introduction}

The provision of evidence-based, clinically effective and efficient wound care out of the hospital environment is a goal that can be achieved through the adoption and deployment of currently available telemedicine technologies. However, the challenge facing the health care system generally and home care clinicians specifically is how to identify and operationalize available telemedicine systems into existing systems of care delivery.

There are a number of synonyms used for the delivery of health care supported by digital technology. Terms such as telematics, telehealth, telemedicine, and e-health are commonly seen in the literature and in the area of wound management. The term TeleWound care has been used at times, however, it may be clearer to refer to these systems generically as telemedicine as this term appears to be the most widely employed.

Telemedicine is defined by the World Health Organization (WHO) as the practice of health care using interactive, visual, and/or data communications. This includes health care delivery, diagnosis, consultation and treatment, as well as education and transfer of medical data. ${ }^{1}$

The introduction of telemedicine technologies is complex, requires significant investment, and their adoption is often dependent on fundamental clinical practice change. These challenges often determine the level of success of the introduction of the new technology and the viability of the change., ${ }^{2,3}$ 
This paper provides a review of the types of telemedicine wound technologies that are currently available, the common design of clinical home wound care systems, and the critical factors that enabled the change to telemedicine to be made while improving clinical outcomes and enhancing patient satisfaction.

\section{Historical management of non-hospital wounds}

Traditionally, home care wound management has not differed substantially from in-hospital care in that wounds have been assessed and documented in similar ways that were employed in the acute setting. Parameters such as wound type, etiology, duration, position, clinical appearance, exudate, wound edges, degree of pain, appearance of surrounding skin, and the presence of signs of potential infection are recorded in the clinical record. ${ }^{4}$

Wound dimensions are also recorded using a variety of methods including the use of centimeter grids to measure surface area, length, width, and the depth of the wound. Tracing the wound outline onto an acetate sheet or even free hand drawing the shape of the wound have been used to determine change in the wound over time. Recording wound dimensions to determine wound healing has been particularly problematic due to the potential for significant measurement error. $^{5}$ Visiting nurses would repeat the documentation of these parameters if there is a change in treatment, the wound deteriorates, or healing does not progress at home visits. In some cases, patients may be required to attend outpatient appointments at specialist multidisciplinary wound clinics or with a general practitioner depending on the nature of the wound and the patient's capacity to travel.

Prior to the review of current wound telemedicine systems it should be noted that ideally a detailed, face to face assessment of the patient and their wound should be conducted by a wound management expert prior to the use of a wound telemedicine system for remote management. This is not always possible, particularly when patients are located in very remote locations; however, a detailed physical assessment and history of the patient is considered an essential component of high quality wound management.

\section{Telemedicine systems available to home care wound clinicians System characteristics}

Wound telemedicine systems that are currently available to home wound care clinicians can be generally divided in two broad groupings and a number of sub groupings based on the mode and combination of communications used.
The first group is commonly termed "store and forward" (SAF) or asynchronous communication systems. ${ }^{6,7}$ In SAF type systems, wound and patient details are recorded at the time of visit, stored, and then forwarded at a later time to an expert/specialist clinician for remote review/consultation. The nature of the data recorded, stored, and later forwarded can be as simple as a digital image of a wound or a more complex data set including wound measurements, characteristics, healing rate, and wound management details can also be recorded. In many cases, SAF type systems are used as an electronic wound medical record rather than being used for remote consultation and treatment support. The way that SAF systems are actually used in day to day clinical care is dependent on the local conditions and the work practices of the home care agencies using the systems. This variation in use is highly dependent on a number of factors such as the nature and structure of the health care system, capabilities of the telemedicine system itself, available information technology infrastructure, geography, the clinical needs of patients, and the time and expertise of the staff using the system.

There are numerous examples of wound management SAF systems in use and they are constantly evolving as technology and communication infrastructure evolves. At the most basic level is the use of digital imaging to record and/or forward wound images. This requires the use of widely available digital cameras and a measurement scale that is included in the frame of the digital image for the purpose of scale. The greatest technical issue with the use of this approach is the ability of the staff to capture a clear, focused, and well lit image of the wound in the patient's home. While this may seem simple it does require some practice and familiarity with the digital camera and its settings. ${ }^{8}$ More sophisticated systems that use digital imaging and computer software to not only record a digital wound image but to enable very precise measurement of the wound, calculation of healing rate, and the capture of wound characteristics include the Advanced Medical Wound Imaging System (Medseed Pty Ltd, Melbourne, VIC, Australia) and The Silhouette Star system (ARANZ Medical, Christchurch, NZ). These systems tend to be used by relatively small groups of clinicians, single health services, and wound researchers whereas the ComCare Mobile system (Silver Chain, Perth, WA, Australia) is used by a very large community nursing service in Western Australia to document, assess, and analyze wound care delivered at the patients' residences. On a larger level, the Western Australian Health Department has deployed the MMEx Wound Management System (WMS; University of Western Australia) throughout the state, an area covering approximately one third of the Australian land mass 
with a widely distributed population that has very remote locations providing wound management. MMEx enables digital imaging, wound analytics, and remote consultation ${ }^{9}$ and it is planned to ultimately link to hospitals, general practice physicians, and community nursing providers.

The second form of wound telemedicine is that of real time video conferencing (VC) or synchronous telemedicine. ${ }^{6}$ In this form, there is a real-time interaction between the clinician and patient with a remote consultant. The requirements of $\mathrm{VC}$ are that all parties are available at the same time to undertake the remote consultation. Once again, the success of $\mathrm{VC}$ is dependent on the availability of communication infrastructure in the patient's home. To date, VC has been better suited to a clinic based interaction with a remote center; however, the gradual introduction of broadband technology to patients' residences will facilitate the potential for greater use of VC in future wound telemedicine.

A third form of wound telemedicine has been described as hybrid systems of SAF and VC. ${ }^{6}$ Here we see the data being forwarded via a SAF system combined with real-time $\mathrm{VC}$ so that detailed data can be reviewed during a synchronous or real-time interaction between the wound care clinician, patient, and remote expert.

\section{Studies of remote wound management, best practice, and new developments in non-hospital wound care}

There are a number of studies investigating the effectiveness of wound telemedicine, however, they are difficult to compare directly due to methodological and technical differences as well as differing local characteristics and service delivery models. Conversely, some common themes do emerge from these investigations. Studies that have evaluated the feasibility of SAF wound telemedicine systems have focused on clinical outcomes such as healing rates, system reliability, financial outcomes, and clinician and patient satisfaction.

A large, randomized controlled trial of the effectiveness of remote wound consultation for patients with chronic lower limb ulcers in the Kimberley region of Western Australia $^{10}$ demonstrated significant clinical and cost benefit of using a SAF wound management system to analyze wound parameters and to conduct remote expert wound consultation. It was found that image quality and clinical data was adequate to enable accurate review and consultation for the complex wounds being treated in four remote locations. Results showed that patients in the telemedicine group had significantly faster healing rates, less amputations, and cost less to treat than controls receiving standard care.
A randomized controlled trial ${ }^{11}$ that compared three groups, two of which received telemedicine interventions and one that received standard care failed to show any benefit (time to heal, cost, and length of stay) of the telemedicine intervention over standard care. This study had significant methodological problems including an unbalanced allocation of patients with more severe wounds to the intervention groups than in the control group.

Clegg et $\mathrm{al}^{12}$ reported an evaluation of a hybrid wound telemedicine system incorporating both SAF and VC technology to undertake expert wound review and reported a saving of approximately US\$100 per visit compared to standard consultation. They concluded that the quality of wound care delivered through the telemedicine system was comparable to face to face consultation. Cost reductions have been found in treating patients at home for chronic wounds ${ }^{13}$ and in home management of leg ulcers using an initial face to face visit followed by a digital image review via a secure website. ${ }^{14}$ Reduction in hospitalization, attendances at emergency departments, and costs have also been reported for patients treated at home by visiting nurses when supported by remote consultation and guideline use via wound telemedicine. ${ }^{15}$

A number of studies have focused on the comparability of the quality of wound assessment using telemedicine systems compared to in-clinic assessment. Central to the success of wound telemedicine systems is the question of accuracy of assessment and clinical efficacy. ${ }^{16}$ In a study on the quality of intra- and inter-rater reliability of clinicians using a three-dimensional (3D) SAF wound telemedicine system, investigators found that the telemedicine system enabled wound assessment accuracy comparable to that of direct consultation for diabetic foot ulcers. ${ }^{17}$ Similar levels of accuracy were reported in an Austrian study on the use of a wound telemedicine system for remote leg ulcer assessment. ${ }^{18}$ Clinicians rated the quality of wound images and reported that in $89 \%$ of 492 consultations the image quality was sufficient to allow them to give therapeutic recommendations remotely. The high degree of clinical assessment accuracy of telemedicine assessment in the home follow-up of pediatric burns patients was reported in an Australian study. ${ }^{19}$

Wound telemedicine systems have also been used in clinical research both in home and clinic settings with ambulant patients. In a large randomized controlled trial of the relative effectiveness of Cadexomere Iodine and nanocrystalline silver dressings for home based patients with colonized venous leg ulcers, a wound telemedicine system was used to measure wound changes and to determine healing rates. ${ }^{20}$ The measurement accuracy of some wound telemedicine 
systems provide significant advantages for wound researchers due to their ability to monitor and measure and quantify discrete changes in wounds such as changes to different tissue types, surface areas, perimeter advancement, and depth within wounds. Additionally, some wound telemedicine systems provide $3 \mathrm{D}$ wound characteristics and analyze healing rates ${ }^{21}$ which may clarify some elements of wound biology and healing.

\section{Patient and carer satisfaction and acceptance of home wound telemedicine}

There appears to be a consistent theme in wound telemedicine literature of clear patient and carer acceptance of this emerging technology. It should be noted that not all wound telemedicine research has investigated patient perceptions as part of the research. Where this element has been incorporated into the research there appears to be different patient responses to SAF technologies than to VC or hybrid type systems, but it is difficult to draw clear conclusions due to methodological differences in these studies. Studies based on SAF approaches report that patients are reassured that their wound is healing due to being able to observe the changes in the wound over time. ${ }^{22,23}$ Binder et $\mathrm{al}^{14}$ reported high levels of patient satisfaction with remote monitoring of home based leg ulcer treatment; this finding is supported in Swedish and Austrian studies ${ }^{24,18}$ on the use of VC for expert consultation for leg ulcer treatment in cohorts of elderly patients. Similar findings of patient satisfaction with $\mathrm{VC}$ are also noted by investigators of VC in the American aged care sector. ${ }^{25}$ Smith et al ${ }^{19}$ also noted high levels of carer satisfaction with wound telemedicine for pediatric patients in remote locations in Australia. One can summarize the limited but positive evidence of patient satisfaction with wound telemedicine as indicating that patients and carers appear to appreciate being involved in their care, monitoring progress with their wounds, and seem grateful for not having to make as many trips to clinical facilities that may involve significant time, inconvenience, and expense. Generally, the topic of patient and carer satisfaction with wound telemedicine is greatly under investigated and should be included in future studies in this area.

\section{Safety, legality, and practicality of remote wound care}

The safety of wound telemedicine systems relates to a number of factors: 1) the performance of the system in terms of comparability to face to face consultation; 2) the validity, reliability, and integrity of measurement performance; and 3 ) the compliance of the wound telemedicine system with national or international wound management guidelines. There is clear evidence of the comparability of wound telemedicine systems with face to face consultation; ${ }^{17,18}$ however, the evolution of commercially available wound telemedicine systems over the past 10 years has not been in all cases accompanied by clear, independently evaluated performance data. The reason for this is that the evolution of these systems has occurred outside of a regulatory framework governing standards for wound telemedicine. Effectively, wound telemedicine has developed through the convergence of clinical need, developing information and communication technology and the availability of low-cost digital photography equipment. The Health Level 7 (HL7; Health Level 7 International, Ann Arbor, MI, USA) international standard for telemedicine defines the interoperability, communication, and security parameters for telemedicine systems, but now there is a need for the development of national/international standards relating to the wound management parameters of wound telemedicine particularly in the area of SAF systems.

The legal position in relation to wound telemedicine is unclear from the perspective of liability of health care organizations for potential harm that may result from the use of these systems particularly in the absence of a regulatory framework and lack of standards for the performance of the systems. ${ }^{26,27}$ Additionally, health care organizations would reasonably be expected to provide adequate training for clinicians to ensure that they are using the equipment appropriately. Patient data integrity and confidentiality is of great concern and should be addressed by organizations ${ }^{28}$ employing wound telemedicine particularly when these systems are not integrated into existing hospital electronic medical records. Unfortunately, it is often the case that these and other issues tend to only be revealed once litigation is initiated.

The practicality of introducing wound telemedicine systems within existing home nursing programs is highly dependent on the characteristics of the service, organizational commitment, case load, funding model and existing information, and communication infrastructure. Due to the potential variability of these and other factors it is not possible to provide definitive answers related to the practicality of introducing these systems; however, it is possible to gain an appreciation of how some services have introduced wound telemedicine and the staff and service response. Senior management support is essential for the introduction of clinical information technology and the effect on the subsequent adoption of the systems across care settings.,29 Similarly, 
clinical staff support is essential to the success of the introduction of these systems. Staff support for telemedicine may vary due to the characteristics and familiarity of staff with wound telemedicine and information technology. ${ }^{30}$ Alternatively, staff may not want to change existing work practices ${ }^{18}$ or may perceive the introduction of wound telemedicine as making existing work practices inefficient. ${ }^{31}$

\section{Discussion}

\section{The place of wound telemedicine in the future of home care practice}

The introduction of wound telemedicine has parallels with the development of many past technologies in that it was driven by the evolution and convergence of other technologies such as personal computers, digital photography, and the internet. Wound digital imaging systems were developed for use in research and to better document and measure wounds in in-patient settings. From these beginnings SAF and $\mathrm{VC}$ wound systems migrated to the community sector and into home care. The drivers for this migration varied from country to country. In some, the evolution was based on cost effectiveness due to the ability to substitute some visits via wound telemedicine and the better, more efficient use of highly skilled staff. The capacity for cost savings also applied to patients due to the reduction in the need for these patients to attend wound clinics while still benefitting from having their wounds reviewed and treatment effectiveness remotely monitored by expert staff.

In other countries, the impetus for the use of wound telemedicine is the need to better serve rural and remote communities that do not have access to specialist wound services. Here patients and local staff benefitted from wound telemedicine due to the elimination of many of the disadvantages of geographical isolation. Expert wound clinicians could provide consultations via SAF technologies and could interact with staff and patients through VC or hybrid systems using both SAF and VC.

A striking aspect of the evolution of wound telemedicine is the lack of standardization and certification of these technologies. It is still possible to buy and commence using a wound telemedicine SAF system today that does not have to comply with any particular technical or clinical standard. This lack of standardization presents a potential risk to both health care organizations and to patients. There is a pressing need for the development of minimum wound care standards for these systems. Similarly, there is no requirement for integration with existing hospital/clinical systems, such as electronic medical records. The use of electronic medical records is by no means universal and this problem is compounded by the "stand alone" nature of a number of SAF wound telemedicine systems. There are some positive signs that this situation is improving: in the home care environment, Silver Chain in Western Australia is a leader in the integration of its ComCare mobile wound telemedicine system with its ComCare electronic patient record. Also in Western Australia, the MMEx system has as part of its design parameters the need to communicate with hospitals and with general practitioners.

While many would ask what the cost/benefit is for developing and deploying wound telemedicine in the home care sector, perhaps it would be more beneficial to ask what the cost would be in the future of not using these systems within countries with aging populations and an increasing burden of chronic disease.

The future of home care wound telemedicine systems will see increasing integration with existing health care organizations. Additionally, we will see the migration of these systems from primarily desktop based systems to mobile devices, such as smart phones and tablets. Within these devices we will also see greater automation of wound analysis functions. Currently, wound management systems require the transfer of an image from a digital camera to a computer for measurement of the wound. This measurement has been undertaken by tracing the margin of the wound so that a surface area measurement can be calculated and used to determine a healing rate. This is a time consuming process and one that will be replaced by automated edge detection technology. This is now available and it can also differentiate between differing tissue types within the wound, measure, and report on their change automatically, thereby making this aspect of wound assessment much more rapid. There are even now prototypes of mobile systems being developed that explore the use of spectral analysis to detect the presence of wound bacteria within a chronic wound.

A further area of development and integration is that of linking wound telemedicine systems with clinical decision support algorithms aligned with evidence based clinical guidelines. Here the aim is to develop a wound telemedicine system that is highly automated in its ability to assess a wound. The system would provide a provisional diagnosis of the wound based on patient history, presenting problems, wound assessment parameters, and would suggest to the clinician a potential course of management based on the most current clinical evidence. Mobile systems would also benefit patients and carers by being able to deliver wound management education applications and in some cases 
wound treatment advice for patients who can self-care for their wounds.

To take this a few steps further there is no reason why electronic patient records could not be linked to all wound telemedicine systems and thereby provide very large databases of wound types, treatment, and healing rates based on treatment. This approach is sometimes termed "big data" but it would enable very robust cost/benefit analyses to be conducted on the effectiveness of various wound treatments regardless of clinical setting.

Home care will always require expert clinical staff to treat patients with wounds and wound telemedicine systems enable these staff to more effectively and efficiently provide high quality care based on the best available evidence.

\section{Disclosure}

The authors report no conflicts of interest in this work.

\section{References}

1. World Health Organization. A Health Telematics Policy in Support of Who's Health for-All-Strategy for Global Health Development: Report of the Who Group Consultation of the Health Telematics, December 11-16, 1997. Geneva: World Health Organization; 1998.

2. Barrett M, Larson A, Carville K, Ellis I. Challenges faced in implementation of a telehealth enabled chronic wound care system. Rural Remote Health. 2009;9(3):1154.

3. Ellis I, Santamaria N, Carville K, et al. Improving pressure ulcer management in Australian nursing homes: Results of the PRIME Trial organisational study. Primary Intention. 2006;14(3):106-111.

4. Carville K. Wound Care Manual. 6th ed. Perth: Silver Chain Foundation; 2012.

5. Santamaria N, Clayton L. Cleaning up. The development of the Alfred/ Medseed Wound Imaging System. Collegian. 2000;7(4):14-15, 17.

6. Chittoria RK. Telemedicine for wound management. Indian J Plast Surg. 2012;45(2):412-417.

7. Ong CA. Telemedicine and Wound Care. In Latifi R, editor. Current Principles and Practices of Telemedicine and E-Health. Washington, DC: IOS Press; 2008:211-225.

8. Jones SM, Banwell PE, Shakespeare PG. Telemedicine in wound healing. Int Wound J. 2004;1(4):225-230.

9. Santamaria N, Glance D, Prentice J, Fielder K. The development of an electronic wound management system for Western Australia. Wound Practice and Research. 2010;18(4):174-179.

10. Santamaria N, Carville K, Ellis I, Prentice J. The effectiveness of digital imaging and remote wound consultation on healing rates in chronic lower leg ulcers in the Kimberley region of Western Australia. Primary Intention. 2004;12(2):62-70.

11. Terry M, Halstead LS, O'Hare P, et al. Feasibility study of home care wound management using telemedicine. Adv Skin Wound Care. 2009;22(8):358-364.

12. Clegg A, Brown T, Engels D, Griffin P, Simonds D. Telemedicine in a rural community hospital for remote wound care consultations. JWound Ostomy Continence Nurs. 2009;38(3):301-304.
13. Dobke MK, Bhavsar D, Gosman A, De Neve J, De Neve B. Pilot trial of telemedicine as a decision aid for patients with chronic wounds. Telemed J E Health. 2008;14(3):245-249.

14. Binder B, Hofmann-Wellenhof R, Salmhofer W, Okcu A, Kerl H, Soyer HP. Teledermatological monitoring of leg ulcers in cooperation with home care nurses. Arch Dermatol. 2007;143(12):1511-1514.

15. Rees RS, Bashshur N. The effects of TeleWound management on use of service and financial outcomes. Telemed J E Health. 2007;13(6): 663-674.

16. Flowers C, Newall N, Kapp S, et al. Clinician inter-rater reliability using a medical wound imaging system. Wound Practice and Research. 2008;16(1):22-31.

17. Bowling FL, King L, Paterson JA, et al. Remote assessment of diabetic foot ulcers using a novel wound imaging system. Wound Repair Regen. 2011;19(1):25-30.

18. Hofmann-Wellenhof R, Salmhofer W, Binder B, Okcu A, Kerl H, Soyer HP. Feasibility and acceptance of telemedicine for wound care in patients with chronic leg ulcers. J Telemed Telecare. 2006; 12 Suppl 1:15-17.

19. Smith AC, Kimble R, Mill J, Bailey D, O'Rourke P, Wootton R. Diagnostic accuracy of and patient satisfaction with telemedicine for the follow-up of paediatric burns patients. JTelemed Telecare. 2004;10(4): 193-198.

20. Miller CN, Newall N, Kapp SE, et al. A randomized-controlled trial comparing cadexomer iodine and nanocrystalline silver on the healing of leg ulcers. Wound Repair Regen. 2010;18(4):359-367.

21. Santamaria N, Ogce F, Gorelik A. Healing rate calculation in the diabetic foot ulcer: comparing different methods. Wound Repair Regen. 2012;20(5):786-789.

22. Austin D, Santamaria N. Digital imaging and the chronic wound: clinical application and patient perception. The Journal of Stomal Therapy Australia. 2002;23(4):24-29.

23. Austin D, Santamaria N. Wound imaging and people with chronic wounds: what happened to hexis? Collegian. 2004;11(3):12-19.

24. Jönsson AM, Willman A. Development of a consultation and teaching concept for leg wound treatment in home health care. $J$ Telemed Telecare. 2007;13(5):236-240.

25. Laflamme MR, Wilcox DC, Sullivan J, et al. A pilot study of usefulness of clinician-patient videoconferencing for making routine medical decisions in the nursing home. J Am Geriatr Soc. 2005;53(8): 1380-1385.

26. Clark PA, Capuzzi K, Harrison J. Telemedicine: medical, legal and ethical perspectives. Med Sci Monit. 2010;16(12):RA261-RA272.

27. Gardiner S, Hartzell TL. Telemedicine and plastic surgery: a review of its applications, limitations and legal pitfalls. J Plast Reconstr Aesthet Surg. 2012;65(3):e47-e53.

28. Gray LC, Armfield NR, Smith AC. Telemedicine for wound care: current practice and future potential. Wound Practice and Research. 2010;18(4):158-163.

29. May C, Harrison R, MacFarlane A, Williams T, Mair F, Wallace P. Why do telemedicine systems fail to normalize as stable models of service delivery? J Telemed Telecare. 2003;9 Suppl 1:S25-S26.

30. Loera JA. Generational differences in acceptance of technology. Telemed $J$ E Health. 2008;14(10):1087-1090.

31. Santamaria N, Austin D, Clayton L. Multi-site trial and evaluation of the Alfred/Medseed Wound Imaging System prototype. Primary Intention. 2002;10(3):119-124. 
Smart Homecare Technology and TeleHealth

Dovepress

\section{Publish your work in this journal}

Smart Homecare Technology and TeleHealth is an international, peer-reviewed, open access online journal publishing original research, reviews, editorials and commentaries on the application of technology to support people and patients at home and in assisted living centers to optimize healthcare and management resources. Specific topics in the journal include: Development and application of

devices within the home and embedded in appliances; Healthcare provider communication and education tools; and drug ordering and adherence. The manuscript management system is completely online and includes a very quick and fair peer-review system, which is all easy to use. Visit http://www.dovepress.com/ testimonials.php to read real quotes from published authors.

Submit your manuscript here: http://www.dovepress.com/smart-homecare-technology-and-telehealth-journal 